Nouveaux outils, nouvelle pédagogie

\title{
Gilberte Furstenberg
}

\section{(2) OpenEdition}

Journals

Édition électronique

URL : http://journals.openedition.org/ries/2922

DOI : 10.4000/ries.2922

ISSN : 2261-4265

\section{Éditeur}

Centre international d'études pédagogiques

\section{Édition imprimée}

Date de publication : 1 juin 1998

Pagination : 87-91

ISSN : 1254-4590

\section{Référence électronique}

Gilberte Furstenberg, " Nouveaux outils, nouvelle pédagogie », Revue internationale d'éducation de Sèvres [En ligne], 18 | juin 1998, mis en ligne le 19 avril 2013, consulté le 19 avril 2019. URL : http:// journals.openedition.org/ries/2922 ; DOI : 10.4000/ries.2922

Ce document a été généré automatiquement le 19 avril 2019

(c) Tous droits réservés 


\title{
Nouveaux outils, nouvelle pédagogie
}

\author{
Gilberte Furstenberg
}

1 La question de savoir s'il faut intégrer l'outil technologique aux méthodes d'enseignement ne se pose évidemment plus, quelle que soit la discipline enseignée ou étudiée. L'école, au sens large du terme, ne peut plus se permettre de rester en marge des développements technologiques, au risque de se couper complètement $\mathrm{du}$ monde extérieur. Elle se doit d'intégrer cette composante, non seulement parce qu'elle existe mais surtout parce qu'elle offre des atouts qui, s'ils sont bien exploités, permettront d'enrichir l'acquisition de connaissances et la compréhension d'un sujet.

2 La grande question est de savoir comment intégrer la technologie de telle sorte qu'elle enrichisse véritablement le savoir des apprenants. C'est celle que se posent maintenant toutes les écoles et universités aux États-Unis comme dans le reste du monde.

3 Cet article rend compte d'expériences pédagogiques dans ce domaine - et plus particulièrement dans le domaine de l'utilisation du multimédia dans le cadre d'un cours de français langue étrangère au MIT ${ }^{1}$. Il ne se propose pas de présenter un "modèle » d'intégration, dans la mesure où les contextes et les objectifs d'apprentissage varient grandement d'une institution à une autre. Il vise seulement à faire part d'expériences et d'éléments de réflexions qui pourraient servir de pistes.

4 Si nous avons procédé au MIT à une réflexion dans ce domaine, c'est en grande partie parce que nous avons utilisé beaucoup d'applications multimédia mais aussi et surtout parce que nous avions nous-mêmes créé des applications pour l'apprentissage du français langue étrangère. Ceci nous a naturellement amenés, voire obligés, à réfléchir sur la démarche la plus appropriée qui permettrait aux enseignants et aux élèves de s'approprier et d'utiliser au mieux cet outil. Cette même réflexion nous a conduits à mettre en évidence les différentes étapes nécessaires pour y arriver. 


\section{De la formation des enseignants...}

5 Il est indispensable, en premier lieu, de familiariser les enseignants avec les outils proposés, que ce soit le cédérom, le Web ou une toute autre technologie. Dans cette formation, doit figurer, avant tout, une compréhension en profondeur de la spécificité et des capacités de chacun de ces outils, qui présente ses propres caractéristiques et atouts. Il faudra donc que les enseignants connaissent ces attributs pour être à même d'en faire une utilisation maximale. Par ailleurs - et fort heureusement - ils n'utiliseront ces nouveaux outils que s'ils en perçoivent la valeur par rapport à leur enseignement ou à l'apprentissage de leurs élèves.

6 Ainsi, la vidéo n'est devenue un instrument privilégié pour l'enseignement et l'apprentissage d'une langue étrangère qu'à partir du moment où les enseignants ont vu et compris l'enrichissement qu'elle pouvait apporter-en l'occurrence sa capacité à ouvrir une fenêtre sur le monde et la culture de la langue enseignée. Encore s'agissait-il d'apprendre à exploiter et utiliser l'image et les indices culturels qu'elle présentait. Cette initiation à l'image, au langage visuel et culturel était (et reste) d'autant plus indispensable que la formation des professeurs est ancrée quasi exclusivement dans le linguistique et le littéraire.

7 L'étape essentielle sera donc de proposer des ateliers de formation où les enseignants pourront s'initier à ces nouveaux outils, manipuler un maximum d'applications, de façon à connaître les notions de base, tels que les différents modes de navigation et les différents degrés d'interaction et d'interactivité. Une familiarisation technique est également essentielle. Un enseignant doit se sentir à l'aise dans l'utilisation des outils technologiques, s'il est amené à les utiliser devant ses élèves. Seule une maîtrise minimale de ces outils (une maîtrise technique parfaite n'est pas nécessaire) pourra inciter un enseignant à intégrer ces outils dans ses cours.

8 Il reste à souligner que ces deux étapes préliminaires tendront à disparaître au fur et à mesure que les nouvelles générations prendront le relais. Le malaise encore éprouvé par les enseignants d'aujourd'hui n'existera bientôt plus.

9 La troisième étape passera nécessairement par une réflexion sur des différents modes d'utilisation de la technologie et posera les questions essentielles : comment l'utiliser, où, quand et par qui ?

10 Il conviendra, d'abord, de faire prendre conscience aux enseignants du fait que les outils technologiques sont conçus, en règle générale, non pas dans la perspective d'un enseignement, mais plutôt d'un apprentissage. Le professeur doit donc savoir non seulement comment les utiliser dans son enseignement mais, peut-être encore plus, comment les faire utiliser par ses élèves. Il ne lui suffira pas de s'approprier ces outils (pour enrichir ses présentations en classe, par exemple) mais il lui incombera d'aider ses élèves à se les approprier (bien que certains en sachent déjà beaucoup plus que leur professeur dans ce domaine). À l'ère de la communication, les objets du savoir, par le biais de la technologie, appartiennent en effet à tous, ou tout au moins à tous ceux qui peuvent y avoir accès, et non plus seulement à l'enseignant.

11 Il faudra alors réfléchir sur les nouvelles pratiques pédagogiques à mettre en place de façon qu'il y ait une véritable adéquation entre les outils, l'apprentissage et l'enseignement. 


\section{... aux nouvelles pratiques pédagogiques}

tâches initiales que les étudiants accompliront individuellement en face à face avec leur
cédérom (que ce soit au laboratoire ou chez eux, selon les possibilités d'accès au produit). Puis, dans un deuxième temps, il conviendra de prévoir, pour la salle de classe, des activités de suivi et de mise en commun au cours desquelles les élèves seront amenés non seulement à échanger leurs informations mais à les rapprocher, à les organiser et à réfléchir collectivement sur le sens ou les sens qui émergent, afin de construire et d'approfondir progressivement leur propre compréhension du sujet. Il ne s'agira donc pas tant d'utiliser l'outil technologique comme mode de présentation par le professeur ou les élèves, mais de mettre plutôt l'accent sur le processus même de la construction d'un savoir par les élèves. La mission de l'enseignant, passant de groupe en groupe, consistera à solliciter des réactions, à faire jaillir de nouvelles questions et, évidemment, à s'assurer de la cohérence (historique, littéraire, culturelle, contextuelle, selon le cas) du raisonnement ou du schéma construit par ses élèves.

Tout ceci bouleverse les rôles traditionnels de l'enseignant et de l'apprenant, ainsi que la dynamique de la salle de classe. Le professeur n'est plus le seul détenteur du savoir - et n'est pas non plus tenu de savoir tout ce que contient un cédérom - ce qui crée une relation beaucoup plus égalitaire entre l'apprenant et l'enseignant. Ce dernier n'est plus là pour dire ou faire dire aux étudiants des choses précises, mais pour les mettre dans des 
situations où ils seront amenés à exprimer, dire, montrer, justifier ce que eux auront vu, lu, entendu et compris.

\section{Des perspectives multiples}

18 Ce type de pratique nous a amenés à observer que les étudiants développent une connaissance et une compréhension plus approfondie et plus intime du sujet (avec souvent une composante affective vis-à-vis des gens, des lieux ou événements rencontrés) et deviennent de plus en plus sensibles à la notion de perspectives multiples, que ce soit celles découvertes au cours de leurs explorations individuelles dans le cédérom ou au cours de leurs interactions avec leurs camarades.

Nous imputons ceci en partie au simple processus de manipulation. Dans la mesure où les étudiants font leurs propres choix, suivent leurs propres itinéraires et peuvent choisir plusieurs perspectives différentes, ils se trouvent au cœur même de l'information, et non plus extérieurs à elle.

20 Par ailleurs, le processus de reconstitution collective d'un contexte, d'un lieu ou d'un événement par le biais des activités de synthèse en classe leur permet de devenir partenaires et constructeurs de leur propre savoir.

21 Cela veut-il dire que la salle de classe elle-même doit se transformer? Certes, elle doit rester le lieu privilégié de la communication par excellence et le lieu où l'étudiant pourra, en confrontant ses idées avec celles de ses camarades et celles du professeur, évaluer la validité des siennes. La salle de classe doit-elle donc être équipée de matériels et de logiciels? C'est certainement souhaitable, mais pas absolument nécessaire. Nous avons remarqué en effet que l'ordinateur agit principalement comme déclencheur et sert en fait de tremplin aux activités collectives. Cela dit, l'idéal est d'avoir dans la salle de classe, non pas au centre, mais à la périphérie, un certain nombre d'ordinateurs qui sont à la portée immédiate de chacun, que ce soit pour revoir une information, la vérifier ou la replacer dans son contexte. L'espace même de la salle de classe s'en trouve changé et la nature de la classe se diversifie, dans la mesure où elle génère plusieurs types d'échanges et permet différents moments et lieux de discussion, de réflexion, de construction ou de synthèse. Un tel arrangement fait que les va-et-vient entre les étudiants, le professeur et l'ordinateur s'en trouvent multipliés et la communication enrichie.

Pour que tout ceci se réalise dans les meilleures conditions, il faut une infrastructure suffisante: des salles de classe équipées et disposant d'un espace adéquat, un soutien technique, l'accessibilité quasi constante aux applications, soit par le biais d'un serveur soit par l'existence de lieux équipés, ouverts de façon quasi permanente. Il est important également que les enseignants soient engagés dès le départ dans les discussions et décisions concernant la mise en place de nouveaux systèmes éducatifs, car ils ont un rôle central à jouer dans ce domaine. Leur imposer la technologie serait de nature à créer une résistance, voire un rejet.

Il reste encore bien sûr toute la question de l'évaluation qui est loin d'être résolue. Chacun, sentant instinctivement qu'un nouvel outil ne peut s'évaluer qu'avec de nouvelles méthodes, est à la recherche de nouveaux modes d'évaluation adéquats. La tendance actuelle, aux États-Unis, va vers la création par les élèves de "dossiers » qu'ils écrivent en continu à travers le semestre ou l'année et où ils relatent à la fois les 
connaissances acquises et une réflexion personnelle sur le processus d'acquisition de ces connaissances.

24 Je crois que nous sommes sur la bonne voie. Personnellement, j'aime extraire le mot «valeur » du mot "évaluation». Si nous pouvons voir et définir clairement la valeur qu'apportent ces nouvelles technologies, la partie est déjà, sinon gagnée, tout au moins bien engagée.

\section{NOTES}

1. Massachusetts Institute of Technology.

\section{RÉSUMÉS}

L'utilisation des nouvelles technologies dans un cours de français langue étrangère au MIT a conduit les enseignants à réfléchir sur l'évolution de leur rôle, sur le mode d'utilisation du multimédia pour l'apprentissage et l'enseignement et, enfin, sur l'évaluation de ces nouvelles formes d'acquisition des connaissances.

INDEX

Index géographique : États-Unis

Mots-clés : formation des enseignants, multimédia, apprentissage du FLE, TICE : technologies de l'information et de la communication en éducation

\section{AUTEUR}

\section{GILBERTE FURSTENBERG}

Senior Lecturer French, Massachusetts Institute of Technology, États-Unis 